\title{
DAMAGE DETECTION IN COMPOSITE CYLINDRICAL PANELS BASED ON FORCED VIBRATION
}

\author{
Marek Barski, Piotr Pająk \\ Cracow University of Technology, Faculty of Mechanical Engineering \\ Jana Pawła II Av. 37, 31-864 Kraków, Poland \\ tel.:+4812 6283389, .+48123743621, fax: +48126283360 \\ e-mail:mbar@mech.pk.edu.pl,ppajak@mech.pk.edu.pl
}

\begin{abstract}
The current work is devoted to the problem of the damage detecting inside the composite cylindrical panels. The applied method is based on the analysis of the forced vibration and corresponding mode shapes of the structure. The presented study has theoretical character (numerical experiment) but it can be easily adopted in practice, for example in case of the SLDV technique. The analysis is performed with use of finite element method. The internal defect is modeled by the reduction of the composite material stiffness and density in arbitrary chosen elements. In the first step the natural frequencies of the intact structure are computed with use of the modal analysis. Next, the corresponding mode shapes are determined (harmonic analysis) for the damage and intact structure. For the each mode shape the curvature in the longitudinal and circumferential direction are determined. Generally, the change of the curvature are very similar in case of damage and intact structure. However, in the location of the damage the change of mode shape curvature are quite different in comparison with intact ones. This fact can be further used in order to detect any kind of flaws inside the analyzed structure. The appropriate damage index is also introduced. Moreover, the simplified analysis of the measurement error influence on the accuracy of the proposed approach is also investigated. The discussed method of the damage detecting in the investigated here multilayered cylindrical panel are very sensitive. Even single small flaws with relatively low reduction of the material stiffness as well as its position can be effectively detected and localized.
\end{abstract}

Keywords: damage detection, forced vibration, natural frequency, composite cylindrical panels, finite element method

\section{Introduction}

The most often observed defects in composite materials are matrix cracking, delamination, fiber breakage or fiber debonding. These defects can arise from various causes: inappropriate manufacturing process, cyclic loads or collision with another object. Moreover, many times those flaws are located inside the material structure. Consequently, the detection of this type of problems at an early stage is very difficult. That is main reason why advanced Non-Destructive Testing methods have to be developed. The description of the most commonly used NDT methods can be found in the work of Hellier [6]. In recent years, methods based on an analysis of the vibration become very attractive. The most important advantage of these approaches is that they can work in on - line mode. In other words, the appropriate equipment is designed as an integral part of the whole structure. If some problem occurs during a service, the warning will be generated automatically. Generally these methods split into two main categories. In the first case the elastic guided waves propagation is analyzed. If a damage exists inside the investigated structure, the received signal will be different in comparison with the intact structured. The type, localization and size of the detected flaw can be estimated with aid of sophisticated computer application. The fundamentals of the wave propagation analysis can be found in the following handbook by Giurgiutiu [4] and Gopalakrishnan et al. [5]. The second approach is based on the fact that if the damage exists the material and structural properties, like natural frequency, mode shape or damping will be changed. Obviously, a magnitude of this change depends on the type, size and location of a defect. It is worth stressing here that now there are available effective experimental 
techniques, which allow to measure the vibration of a structure with high accuracy. One of them is a Scanning Laser Doppler Vibrometer (SLDV). Moreover, the results, obtained from the SLDV, can be very easily simulated by the finite element method. This fact significantly simplify the process of the elaboration an appropriate algorithm of damage detection in the investigated structure. A comprehensive review of the research in this area over last 30 years can be found in work by Doebling et al. [3]. The newest trends in damage detection are discussed in the book by Ostachowicz et al. [10].

Unfortunately, in case of different composite structures the change of the natural frequency due to the existence of a damage is not significant $[1,7,9]$. In practice it means that the damage has to be relatively large to be detectable in this way. Moreover it seems that the precise estimation of a location of a flaw is difficult or even impossible. Thus it is necessary to analyze additional modal parameters, like mode shape or mode shape curvature. Generally, in order to detect a flaw it is necessary to compare the appropriate mode shape of structures damaged and undamaged. The mode shape of the intact structure can be obtained from measurement or from FE analysis. This technique is known as the Modal Assurance Criterion (MAC) [5]. There are various approaches, which utilizes this idea. They differ to each other with details.

However much more sensitive are the techniques based on an analysis and comparison of a mode shape curvature of damage and intact structure. Pandey et al. [11] in 1991 proposed the method of damage detection, where the curvature is computed with aid of the central difference schema. The mode shape was obtained from the numerical study of a dynamic response of a structure. Their results showed that the change of mode shape curvature is localized around the defect and it can be used in order to estimate the localization and extend of the flaw. However, the main disadvantage of the damage detection methods based on the vibration and mode shape is that they are susceptible on noise caused by measurement errors. Thus new concepts of the damage indicator or advanced method of a signal processing are proposed. The damage index based on a strain energy distribution for damage and intact structure are considered by Kim et al. [8] in case of beams and by Cornwell et al. [2] in case of plate-like structures. Furthermore, Radzieński et al. [12] introduced a new method of the SLDV experimental data processing. The obtained results effectively shows the location and extent of the damage in case of steel cantilever beam with a notch.

\section{Composite panel}

In the Fig. 1 the considered intact panel is depicted. The total length of the structure is equal to $\mathrm{Lp}=310[\mathrm{~mm}]$, the width $\mathrm{Wp}=180[\mathrm{~mm}]$ and the radius of the curvature $\mathrm{Rp}=92[\mathrm{~mm}]$. The total thickness is equal to $\mathrm{tc}=2[\mathrm{~mm}]$. The composite material consists of $\mathrm{N}=8$ layers. The play orientations are of $\left[90^{\circ}, 0^{\circ}, 90^{\circ}, 0^{\circ}, 90^{\circ}, 0^{\circ}, 90^{\circ}, 0^{\circ}\right]$. The play orientation angle is measured with respect to the axis of the cylinder. The material constants, taken from [1] are as follows $E_{1}=125$ $[\mathrm{GPa}], \mathrm{E}_{2}=8.5[\mathrm{GPa}], \mathrm{G}_{12}=4.5[\mathrm{GPa}], v_{12}=0.3$ and the density $\rho=1550\left[\mathrm{~kg} / \mathrm{m}^{3}\right]$. Each layer has the same thickness, namely $\mathrm{t}=\mathrm{t}_{\mathrm{c}} / \mathrm{N}$. One edge is fixed and the rest are freely supported. The structure is subjected to the point load (shaker). The location of the load is determined by the dimension $Z_{\mathrm{f}}=78$ $[\mathrm{mm}]$ and its value is equal $\mathrm{F}=0.5[\mathrm{~N}]$.
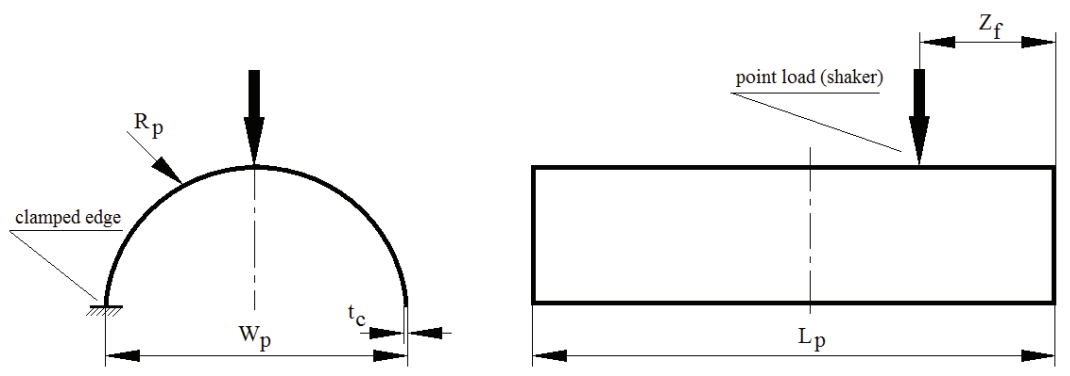

Fig. 1. Composite cylindrical panel 
The damage is located in the geometrical center of the structure and is modeled as rectangular area, where the material stiffness is reduced. The magnitude of the damage is determined by the coefficient $\beta$. Here it is assumed that $\beta=0.85$. It means that the material inside the damage is "weaker" about $15 \%$ in comparison with rest of the composite material. In the Fig. 2 there is shown the dimension of the damage. They are equal to $L_{d}=7.4[\mathrm{~mm}]$ and $W_{d}=5.8[\mathrm{~mm}]$. The dimension $\mathrm{W}_{\mathrm{d}}$ should be consider as the length of the are in the circumferential direction.

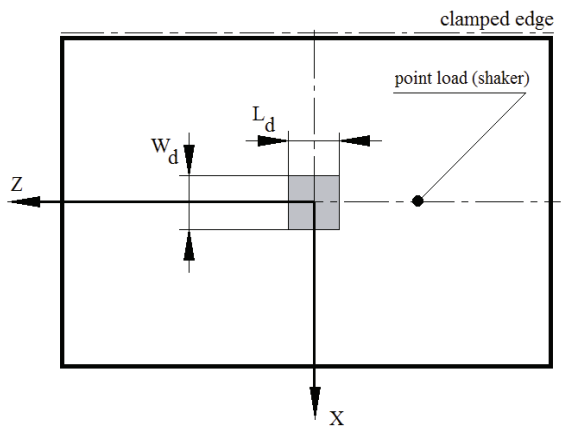

\section{Method}

Fig. 2. Dimensions of damage

The applied here method is based on the comparison of the mode shape curvature of the defected and intact structure. This comparison is made for the several following values of natural frequencies, which are determined for the intact structure. It is assumed that the flaws are sufficiently small. Thus the differences of the natural frequencies computed for the intact and defected structure are negligible. In the first step of analysis the first several values of the natural frequencies of the intact structure have to be determined. This can be done with aid of the finite element method and the modal analysis. Next, in the neighborhood of each evaluated natural frequency value the harmonic analysis is carried out. This FE analysis is performed for damaged and intact structure. As a result the mode shapes described in the cylindrical coordinate system are obtained. In the current analysis only the radial component of the displacement is taken under consideration. The appropriate results are then exported from the FE package as a text file containing the radial displacement components of the mode shapes. Now the change of the curvature is computed. It can be done with use of the central finite difference formula for the circumferential and longitudinal direction respectively:

$$
\kappa_{i, j}^{\theta}=\frac{\partial^{2} U_{i, j}^{(r)}}{\partial r^{2}}=\frac{1}{R_{p}{ }^{2}} \frac{U_{i-1, j}^{(r)}-2 U_{i, j}^{(r)}+U_{i+1, j}^{(r)}}{(\Delta \theta)^{2}}, \kappa_{i, j}^{Z}=\frac{\partial^{2} U_{i, j}^{(r)}}{\partial z^{2}}=\frac{U_{i, j-1}^{(r)}-2 U_{i, j}^{(r)}+U_{i, j+1}^{(r)}}{(\Delta z)^{2}},
$$

where $i=1,2, \ldots, n_{c}, j=1,2, \ldots n_{r}$. The $n_{c}, n_{r}$ denotes the number of finite elements in the circumferential and longitudinal direction. In the surroundings of the panel edges the missing values of $\kappa_{\theta}$ and $\kappa_{Z}$ can be extrapolated. Here the square interpolation is used. Furthermore, the mode shape curvature criterion (MSC) can be introduced in the following matrix form, namely:

$$
C_{i, j}^{\theta}=\left|\kappa_{i, j}^{\theta D A M A G E}-\kappa_{i, j}^{\theta I N T A C T}\right|, C_{i, j}^{Z}=\left|\kappa_{i, j}^{Z \text { DAMAGE }}-\kappa_{i, j}^{Z I N T A C T}\right| .
$$

Moreover, it is convenient to normalize the above criterions with respect to the maximal value, It is worth stressing the further analysis can be carried out in each direction independently. As it is reported in literature [5], in particular mode shapes the damage do not significantly affect the mode shape curvature. This effect is especially visible, when the flaw is located in the area where the strain energy of the deflected structure reaches minimum. Thus it is necessary to analyze a several different mode shapes. Finally, the information about the damage existence, its location and extent can be summarized by the average index, namely: 


$$
I_{i, j}^{A V R}=\frac{1}{m} \sum_{k=1}^{m}\left[\left\{\frac{C_{i, j}^{\theta}}{\max \left|C_{i, j}^{\theta}\right|} \cdot \frac{C_{i, j}^{Z}}{\max \left|C_{i, j}^{Z}\right|}\right\}^{(k)}\right],
$$

where $\mathrm{m}$ is the number of the mode shapes, which are taken into account. It is worth stressing here that the most important advantage of the discussed above approach is its simplicity. Moreover it can be also adopted in case of the structure with double arbitrary curvature.

\section{Results of simulations}

In order to carry out the numerical simulation the commercially available FE package ANSYS ver. 12.1 is used. The quadrilateral (four nodes) shell element SHELL181 is applied. These elements has 6 Degrees of Freedom (DOF) in each nodes, namely the displacement DOF (UX, UY, UZ) and the rotational DOF (ROTX, ROTY, ROTZ). The element formulation is based on the First Order Shear Deformation theory. Moreover, they are especially suitable for modeling of multi-layered composite materials. Further computations are performed with aid of the own program, which is created in VISUAL $\mathrm{C}++2005$. The all diagrams are made in other commercial graphical applications.

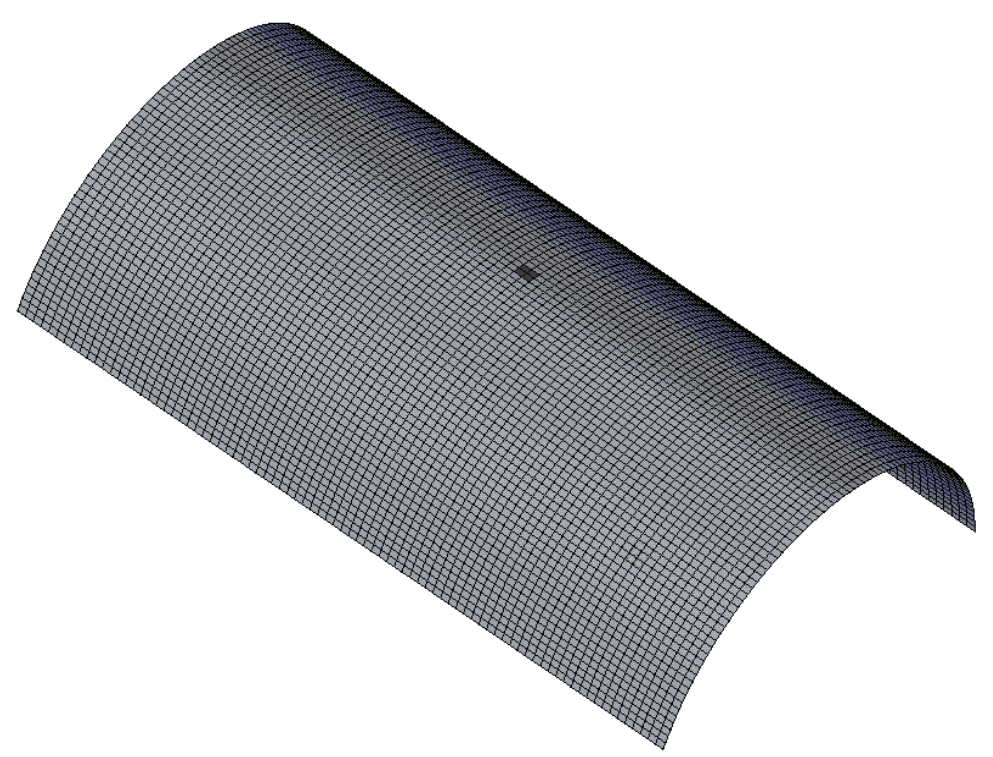

Fig. 3. Finite element mesh with visible damage

\subsection{Modal analysis}

In the first step of the presented analysis the natural frequencies of the investigated structure are determined. Here it is assumed that there are no any material damping. The Block Lanczos mode-extraction method is used. Moreover, the convergence of the finite element results are also tested. It is assumed that the finite element mesh is very regular, so called mapped mesh. Due to the long time of further computations, the finite element mesh with the number of elements equal to $\mathrm{Ne}=7224$ is used. In the Fig. 3 the assumed finite element mesh, created for the damaged panel is depicted. The modal analysis is carried out also in case of the damaged panels and the obtained natural frequencies are stored in the Tab. 1. As it is expected, in case of the damaged structures the change of the appropriate values of natural frequencies are not significant and it should be rather treated as a numerical error. Generally, the existence of a material defect causes the reduction of the values of the natural frequency. This effect is partially observed in the case of all panels but in some cases slight increasing of this value is found. Taking under consideration a measurement 
error and variable environmental conditions (temperature, humidity), the change of the natural frequency is not suitable method of the damage detection. Moreover, a flaw or flaws have to be considerably larger to affect the natural frequencies in the reasonable way.

Tab. 1. Natural frequencies [Hz] of studied panels

\begin{tabular}{|c|c|c|}
\hline Mode & Intact Panel & Damaged Panel \\
\hline 1 & 38.671 & 38.671 \\
\hline 2 & 44.787 & 44.786 \\
\hline 3 & 128.905 & 128.906 \\
\hline 4 & 136.188 & 136.180 \\
\hline 5 & 431.564 & 431.567 \\
\hline
\end{tabular}

\subsection{Harmonic analysis and damage detection}

In order to simulate the real experiment the harmonic response analysis is carried out. The necessary modal shape data are estimated in the close vicinity of the natural frequencies presented in the Tab. 1. The frequency step is assumed to be equal $v_{\text {step }}=0.5[\mathrm{~Hz}]$. It means, for example, that the first modal shape (corresponding to the fundamental natural frequency) is obtained for frequency $v=38.5[\mathrm{~Hz}]$. The full solution method is used. Moreover, the global damping is taken into account and the global damping ratio is equal to $\xi=0.005$. This small value of the damping ratio makes that the numerical analysis to be more realistic and doesn't significantly affect the values of the natural frequency values determined in the modal analysis.

In the Fig. 4, 5 and 6 the curvatures in the circumferential and axial direction of the considered panels are depicted. These diagrams are made along the following lines $Z=0$ and $X=0$, so they pass thought the center of the flaw. Moreover, they are prepared for the following natural frequencies, namely: $38.5[\mathrm{~Hz}], 45[\mathrm{~Hz}]$ and $129[\mathrm{~Hz}]$. These values correspond to the first three modes of the free vibrations. In the third case the damage is localized in the area, where the strain energy reaches the maximum, hence the local change of the curvature is the most visible. However, it should be stressed that the disturbance is not significant. It should be stressed that in the real experiment these local changes would be difficult to detect. Quite different situation is observed in the case of the axial curvature. Here two main disturbances can be observed. First of them is caused by the shaker and the second is connected with existence of the flaw. The position of the damage is clearly visible, especially in the Fig. 4. It seems that the observed local disturbance of the curvature in the axial direction gradually decays for the higher values of the natural frequency. In the Fig. 7 the final damage localization, according to the formula (3), is shown. The position and the size of the damage is clearly visible.
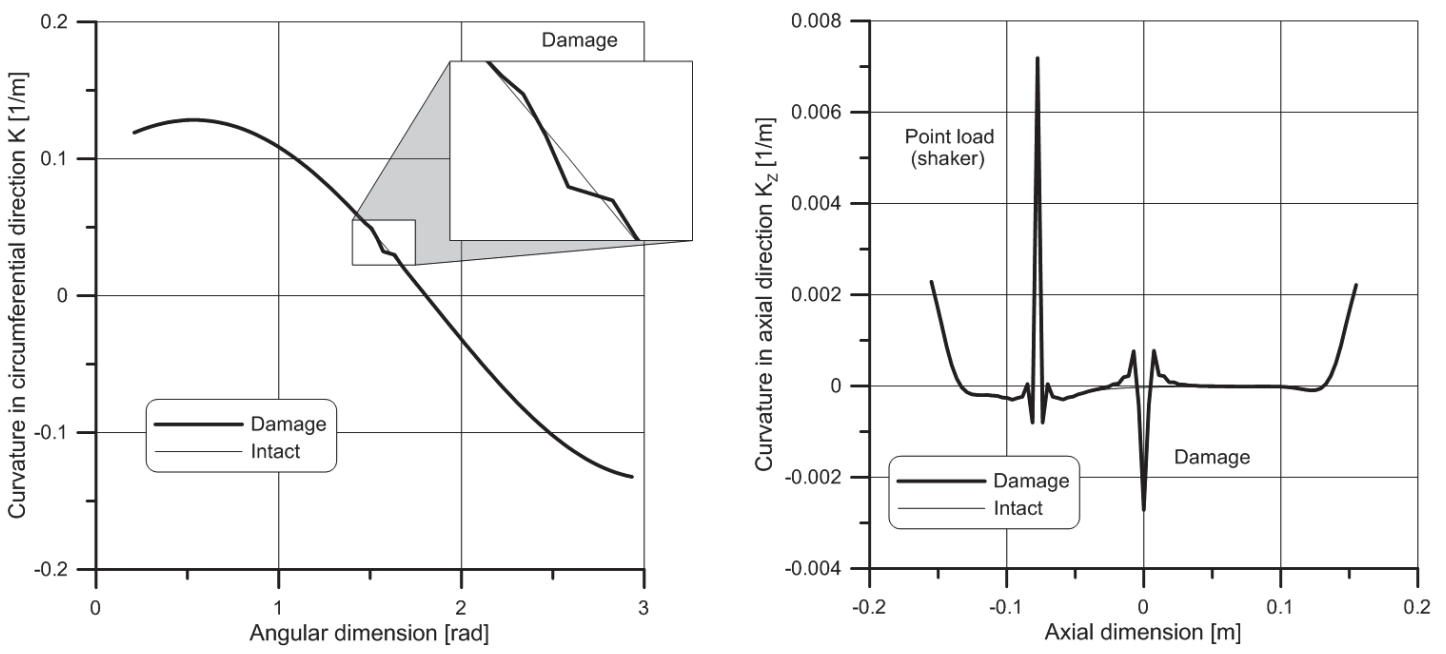

Fig. 4. Curvatures of the panels (description in text). First mode v=38.5 [Hz] 

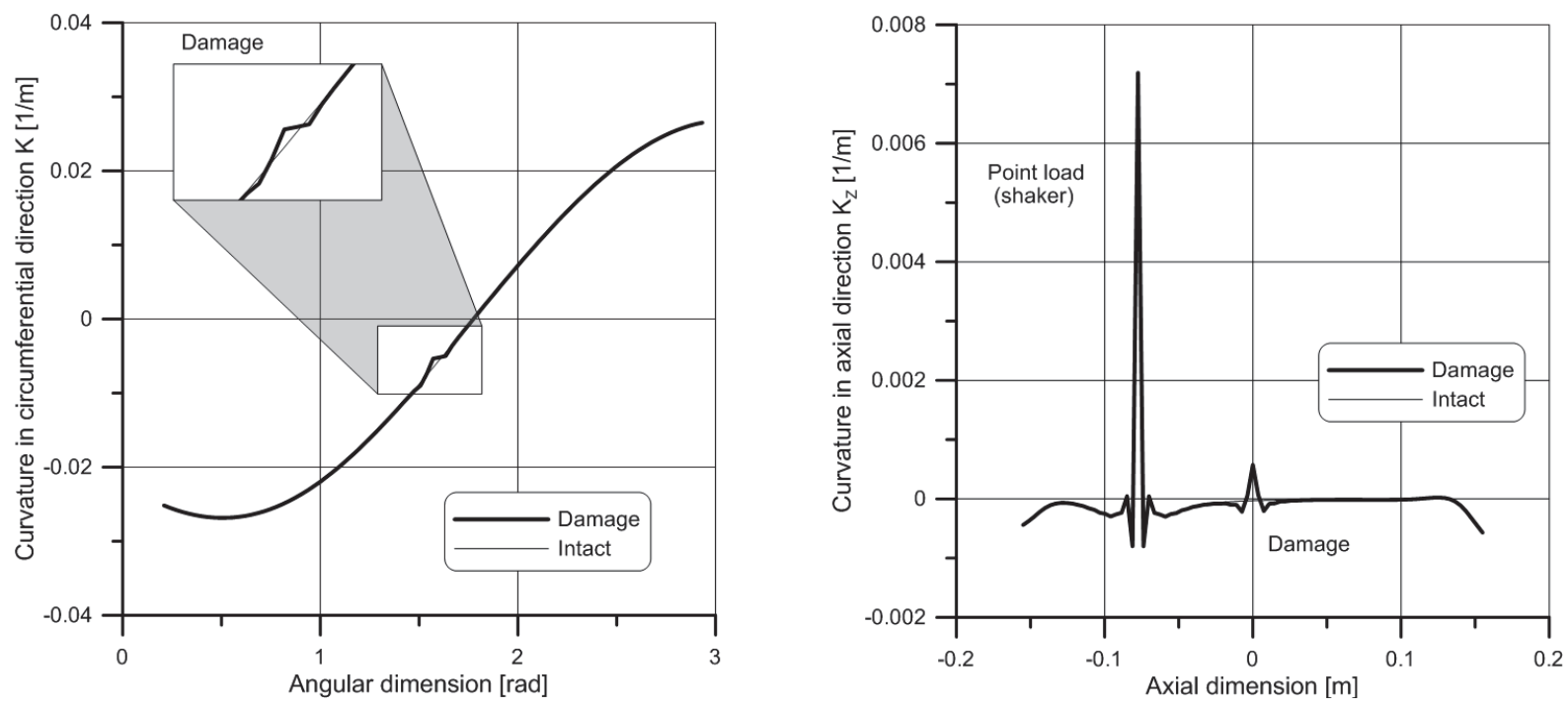

Fig. 5. Curvatures of the panels (description in text). First mode $v=45[\mathrm{~Hz}]$
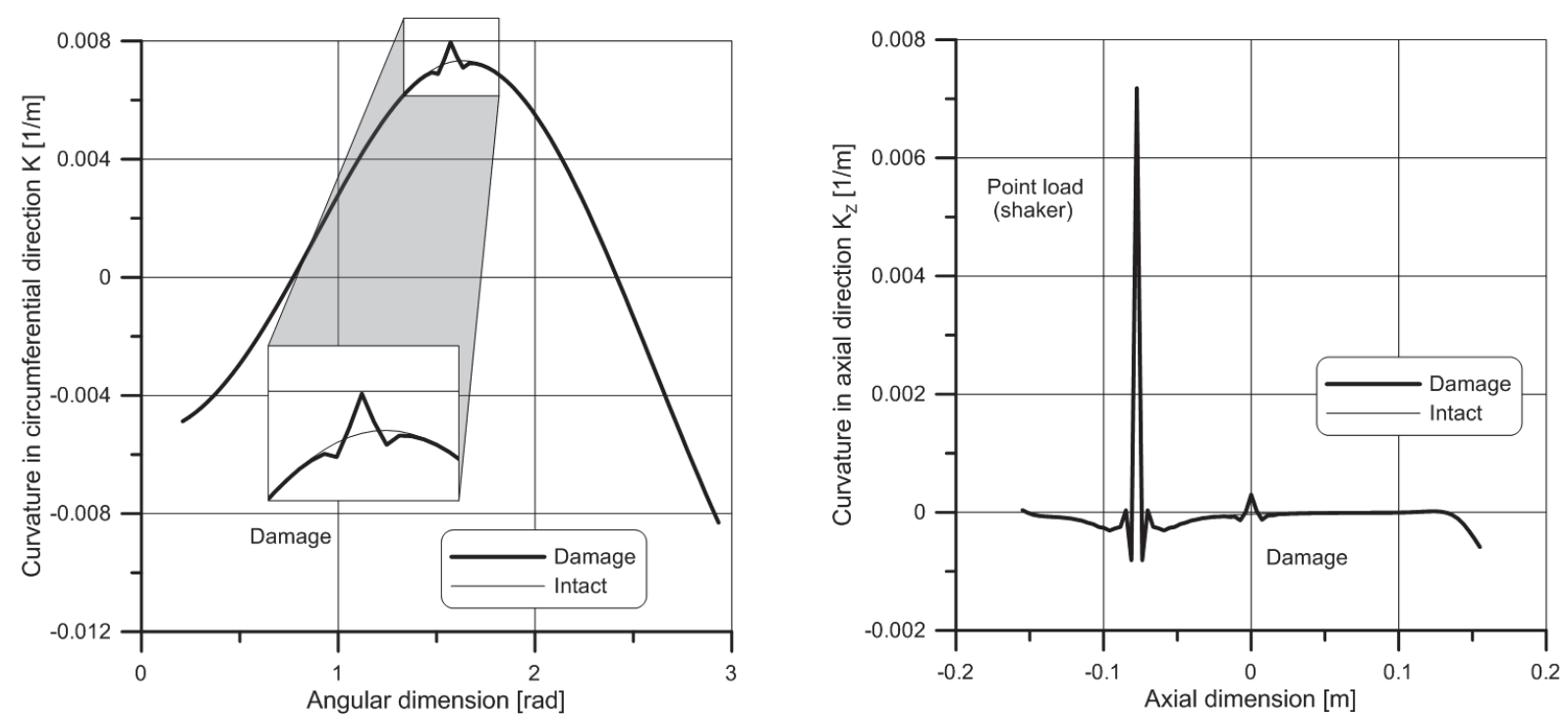

Fig. 6. Curvatures of the panels (description in text). First mode $v=129[\mathrm{~Hz}]$

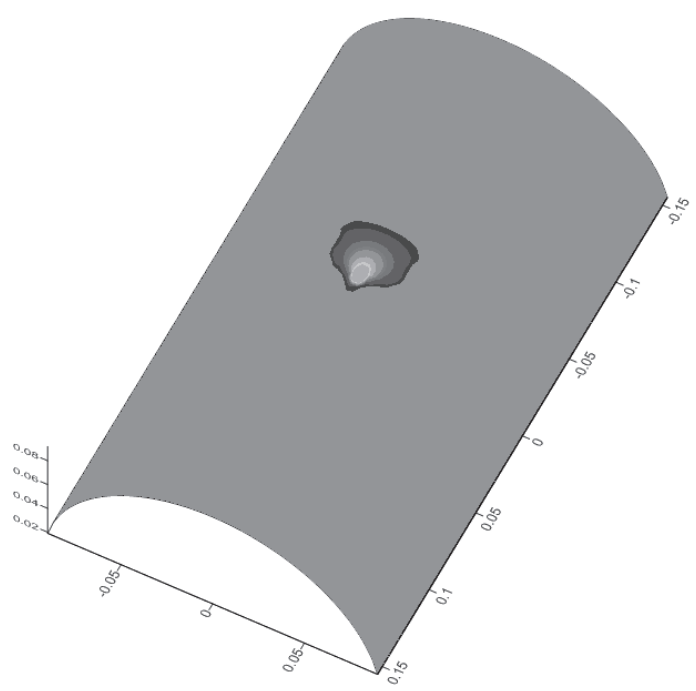

Fig. 7. Analysed panel with visible damage 


\subsection{Influence of measurement error}

In order to simulate the measurement error the radial component of the deflection in each node is randomly disturbed according the following formula:

$$
\widetilde{U}_{i, j}^{(r)}=U_{i, j}^{(r)}+\Delta U(2 \xi-1), \Delta U=0.5\left|U^{(r) \max }-U^{(r) \min }\right| \eta,
$$

where $\xi$ is the random number from $[0,1]$. Moreover, the magnitude of the disturbance depends on the minimal $\mathrm{U}^{(\mathrm{r}) \min }$ and maximal $\mathrm{U}^{(\mathrm{r}) \max }$ value of the radial component of the deflection and arbitrary chosen factor $\eta$. For example, in the case of the first mode $v=38.5[\mathrm{~Hz}]$, the mentioned quantities are equal to $\mathrm{U}^{(\mathrm{r}) \min }=-1.2385958 \mathrm{e}-3[\mathrm{~m}]$ and $\mathrm{U}^{(\mathrm{r}) \max }=0.0[\mathrm{~m}]$. It occurred that if the factor $\eta$ is great than approximately $\eta>0.07$ the flaw will be very difficult to detect. Thus it is necessary to develop the advanced algorithms of the experimental data processing [12]. Below, in the Fig. 8, there is presented the considered panel in case when the factor $\eta=0.065$. As it can be observed, the flaw is still visible.

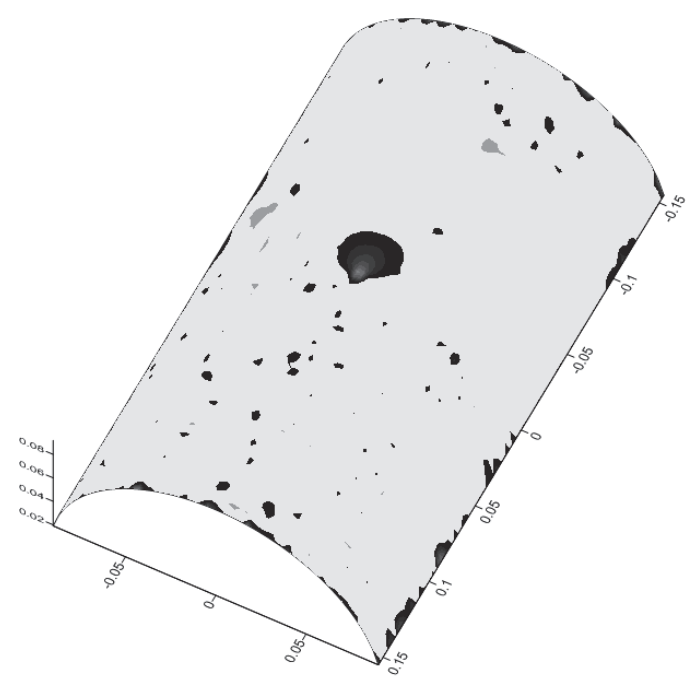

Fig. 7. Analysed panel with visible damage and measurement error taken into account $\eta=0.065$

\section{Conclusion}

The presented here method in the case of the composite cylindrical panels seems to be very effective. Even the small flaws at the early stage of the evolution, like matrix cracking, fibre breakage or delaminations, can be detected with aid of the proposed method. It is worth stressing here that all of the mentioned forms of damage cause the reduction of the density and stiffness of the composite materials. At the very beginning of the damage formation the stiffness reduction of a composite material is not significant. However this small local effect has a radical influence on the shape of the curvature. This fact can be used in order to detect the existence of the defect, which is not externally visible. Now it seems to be impossible to determine the type of damage, which has been detected. Only the extent and the position can be successfully estimated with use of the presented method. The effectiveness of this method is also determined, in practice, much depends on the sensitivity of the test equipment.

\section{References}

[1] Alnefaie, K., Finite element modeling of composite plates with internal delamination, Composite Structure, Vol. 90, pp. 21-27, 2009. 
[2] Cornwell, P., Deobling, S. W., Farrar, C. R., Application of the strain energy damage detection method to plate-like structures. Journal of Sound and Vibrtion, Vol. 224(2), pp. 359-374, 1999.

[3] Doebling, S. W., Farrar, C., Prime, M. B., Daniel, W. S., Damage identification and health monitoring of structural and mechanical systems from changes in their vibration characteristic: a literature review, LA - 13070-MS, May, 1996.

[4] Giurgiutiu, V., Structural Health Monitoring with piezoelectric wafer active sensors, Elsevier, Amsterdam, Boston 2008.

[5] Gopalakrishnan, S., Ruzzene, M., Hanagud, S., Computational Techniques for Structural Health Monitoring, Springer, Dordrecht, Heidelberg, New York 2011.

[6] Hellier, C., Handbook of Nondestructive Evaluation, McGraw-Hill, New York 2001.

[7] Kessler, S. S., Spearing, M. S., Atalla, M. J., Cesnik, C. E., Soutis, C., Damage detection in composite materials using frequency response methods, Composites: Part B, Vol. 33, pp. 87-95, 2002.

[8] Kim, J. T., Stubbs, N., Crack detection in beam type structures using frequency data. Journal of Sound and Vibration, Vol. 259(1), pp. 146-160, 2003.

[9] Kim-Ho, Ip, Ping-Cheung Tse, Locating damage in circular cylindrical composite shells based on frequency sensitivities and mode shapes, European Journal of Mechanics A/Solids, Vol. 21, pp. 615-628, 2002.

[10] Ostachowicz, W., Güemes, J.A., New Trends in Structural Health Monitoring, International Centre for Mechanical Sciences, Courses and Lectures, Vol. 542, CISM, Udine, Springer, 2013.

[11] Pandey, A. K., Biswas, M., Samman, M. M., Damage detection from changes in curvature mode shapes, Journal of Sound and Vibration, Vol. 145(5), pp. 321-332, 1991.

[12] Radzieński, M., Krawczuk, M., Palacz, M., Improvement of damage detection methods based on experimental modal parameters. Mechanical Systems \& Signal Process, Vol. 25, pp. 21692190, 2011. 